\title{
EXECUTIVE CONTROL OF THOUGHT AND ACTION *
}

\author{
Gordon D. LOGAN
}

Purdue University, USA

Accepted July 1985

In addition to processing information about stimuli in the environment, the cognitive system performs executive functions to control and coordinate the processes that deal with environmental stimuli. Several executive functions may be distinguished: (1) Making choices about alternative strategies for processing environmental stimuli; (2) constructing or instantiating a version of the chosen strategy to enable performance on the task; (3) controlling and coordinating execution of the strategy during real-time performance on the task; and (4) disabling or disengaging the strategy in response to changes in goals or changes in the task environment that make the current strategy inappropriate. This paper presents a selective review of research addressing each of these executive functions, focusing on the time and resources used to accomplish each one.

\section{Introduction}

Most theory and research in cognitive psychology is concerned with processes that deal with information about the environment, proposing how environmental stimuli might be encoded, recognized, transformed, represented in memory, and retrieved from memory. Mental processes are often studies in isolation, with little attention paid to how they might work together in complex tasks. The underlying idea is that separate theories of individual processes can be fit together like the

* This review was presented at the symposium on Action, Attention, and Automaticity, held at the Center for Interdisciplinary Studies (ZiF) at the University of Bielefeld, November, 1984.

I am grateful to Jane Zbrodoff for helping to shape and develop the ideas presented here, to Daniel Kahneman and Anne Treisman for helping to clarify them, and to Odmar Neumann for editorial assistance in preparing the article.

Requests for reprints should be addressed to G.D. Logan, Dept. of Psychological Sciences, Purdue University, West Lafayette, IN 47907, USA. 
pieces of a jigsaw puzzle to describe the working of the entire cognitive system, as if the whole were no more than the sum of its parts. However, significant problems of control and coordination may emerge when the separate processes must work together, and it may not be possible to anticipate these problems by studying the processes in isolation. The whole may be more than the sum of its parts.

My research for the past few years has focused on these emergent problems, investigating the executive functions that the cognitive system employs to deal with them. I have investigated four major executive functions (there may be more or less than four; the list is intended as a heuristic to guide research, not as a formal delineation of the possibilities): (1) Choice among different strategies for performing a task, (2) construction or instantiation of the chosen strategy to enable performance of the task, (3) execution and maintenance of the strategy to perform the task, and (4) inhibition or disablement of the strategy in response to changes in goals or changes in the task environment. The purpose of this article is to review selectively the research on each of these executive functions, focusing primarily on my own work.

\section{Choosing a strategy}

Many of the tasks we face in everyday living can be performed in several ways. Each alternative approach to a task constitutes a strategy. More formally, a strategy may be defined as an optional organization of cognitive resources or abilities that is designed to achieve some goal in some task environment (Logan and Zbrodoff 1982; Logan et al. 1983). Since several strategies could be used for most tasks, choice among strategies is an important aspect of everyday mental life.

Jane Zbrodoff and I investigated strategy choice by allowing subjects some options for performing experimental tasks while we manipulated factors that might affect the option they choose. Our experiments focused on a version of the Stroop task, in which subjects reported the identity of a word (the word ABOVE or BELOW) presented above or below a fixation point by pressing keys. (For illustration, we assume subjects pressed the right kcy to indicate ABOVE and the left key to indicate BELOW. In the actual experiments, stimulus-to-response mapping was counterbalanced across subjects.) 
Subjects had three strategic options in performing this spatial Stroop task:

(1) They could attend selectively to the identity of the word, trying to ignore its position, which can be represented by the following productions:

W1: IF word identity = ABOVE, THEN press the right key.

W2: IF word identity $=$ BELOW, THEN press the left key.

This strategy would produce responses that were accurate but relatively slow (the display conditions were such that subjects required about $500 \mathrm{msec}$ to respond to the word's identity but only $350 \mathrm{msec}$ to respond to the word's position).

(2) Subjects could attend selectively to the position of the word, ignoring its identity. If position and identity were compatible (e.g., ABOVE above fixation and BELOW below fixation), subjects could apply the following productions:

P1: IF position $=$ above, THEN press the right key.

P2: IF position $=$ below, THEN press the left key.

If position and identity conflicted (e.g., ABOVE below fixation and BELOW above fixation), subjects could apply the following productions:

P3: IF position $=$ above, THEN press the left key.

P4: IF position $=$ below, THEN press the right key.

$\mathrm{P} 1$ and P2 would produce fast, accurate responses every time position and identity were compatible but would produce errors every time position and identity conflicted, whereas $\mathrm{P} 3$ and $\mathrm{P} 4$ would produce fast, accurate responses every time position and identity conflicted but would produce errors every time position and identity were compatible. (3) Subjects could divide attention between position and identity, priming the response to the word's identity once they knew its position. If compatible stimuli were more frequent than conflicting stimuli, subjects could apply the following productions in combination with W1 and W2:

D1: IF position $=\approx$ above, THEN prime right response.

D2: IF position $=$ below, THEN prime left response. 
D1 and D2 would produce relatively fast, accurate responses when position and identity were compatible and would produce slow, inaccurate responses when position and identity conflicted, enhancing the usual Stroop effect. If conflicting stimuli were more frequent than compatible stimuli, subjects could apply the following productions in combination with $\mathrm{W} 1$ and $\mathrm{W} 2$ :

D3: IF position $=$ above, THEN prime left response.

D4: IF position $=$ below, THEN prime right response.

D3 and D4 would produce relatively fast, accurate responses when position and identity conflicted and would produce slow, inaccurate responses when position and identity were compatible, reducing and perhaps reversing the usual Stroop effect.

Obviously, subjects who were trying to maximize speed and minimize error rate (i.e., trying to obtain the instructed goals) would choose strategy (1), employing productions W1 and W2. Strategy (2) is appropriate only if all the stimuli were compatible or all the stimuli conflicted, and strategy (3) is appropriate only if compatible stimuli predominate or if conflicting stimuli predominate. We attempted to induce subjects to rely on strategies (2) and (3) by manipulating the relative frequency of compatible and conflicting stimuli, introducing a correlation between position and identity so that subjects could predict the word's identity by knowing its position.

In a number of experiments, we found evidence that subjects adopted strategy (3), employing productions D1-D4: As the relative frequency of compatible stimuli increased from 50\% (independence) to $90 \%$ (high correlation), subjects became progressively faster in responding to compatible stimuli and progressively slower in responding to conflicting stimuli, exaggerating the usual Stroop effect. As the relative frequency of conflicting stimuli increased from 50\% (independence) to $90 \%$ (high correlation), subjects became progressively faster in responding to conflicting stimuli and progressively slower in responding to compatible stimuli, attenuating and actually reversing the usual Stroop effect (Logan and Zbrodoff 1979). We have since replicated these findings with other versions of the spatial Stroop task (Logan 1980a), with the usual color-word Stroop task (Logan et al. 1984b), and with an arithmetic version of the Stroop task (e.g., verifying equations such as $3+4=12$ and $3 \times 4=7$; Zbrodoff and Logan in press). In all cases, 
the magnitude of the Stroop effect increased as the frequency of compatible stimuli increased and decreased as the frequency of conflicting stimuli increased, though we were unable to reverse the color-word version of the Stroop effect [1].

It is important to note that subjects gained speed by adopting the divided attention strategy without sacrificing accuracy; they were able to respond faster to the most frequent type of stimulus. Thus, the experiments demonstrate that subjects will adopt strategies that allow them to maximize the attainment of their goals, in this case, responding quickly and accurately. The experiments suggest that cue validity is an important determinant of strategy choice: Subjects relied on the strategy more the stronger the correlation between the dimensions of the Stroop stimuli. The stronger the correlation, the more likely they were to gain speed and accuracy by dividing attention and the less likely they were to lose speed and accuracy. We might expect this principle to extend beyond the limits of the simple Stroop situations Zbrodoff and I studied. Indeed, there is evidence that cue validity is an important determinant of strategy choice in priming studies (see Neely 1977; Posner and Snyder 1975; Tweedy et al. 1977). Other determinants of strategy choise remain to be discovered. Costs and benefits in terms of resource demands are a likely possibility (Logan et al. 1983).

\section{Strategy construction}

Once an appropriate strategy is chosen, it must be constructed or instantiated somehow. The process of strategy construction, like any other process, must take time and consume resources. Thus, it can be studied by investigating the time it requires and the resources it consumes.

Jane Zbrodoff and I have addressed these issues in our spatial Stroop task (Logan and Zbrodoff 1982; Logan et al. 1983). In our studies, the

[1] We may not have been able to reverse the color-word Stroop effect because it was substantially stronger than the spatial Stroop effect, averaging $116 \mathrm{msec}$ (Logan et al. 1984b: experiment 2) while the spatial Stroop effect averaged $20 \mathrm{msec}$ (Logan and Zbrodoff 1979: experiment 1). Thus, the strategies in the color-word task had more of a Stroop effect to overcome than the strategies in the spatial task. Indeed, the strategic effects were about the same magnitude in the two tasks: increasing the relative frequency of conflicting trials from $20 \%$ to $80 \%$ reduced the Stroop effect by $120 \mathrm{msec}$ in the color-word task (Logan et al. 1984b: experiment 2) and by 123 msec in the spatial task (Logan and Zbrodoff 1979: experiment 1). 
four different stimuli (i.e., ABOVE above fixation, BELOW below fixation, ABOVE below fixation, and BELOW above fixation) occurred equally frequently, but subjects were informed of the relation between the dimensions of the upcoming stimulus before each trial: We presented an $\mathrm{X}$ before each compatible stimulus (i.e., ABOVE/above and $\mathrm{BELOW} /$ below) and an $\mathrm{O}$ before each conflicting stimulus (i.e., ABOVE/below and BELOW/above). On these cue valid trials, subjects could attend selectively to the word's position, employing strategy (2) as described above, using productions $\mathrm{P} 1$ and $\mathrm{P} 2$ if $\mathrm{X}$ was the cue and productions $\mathrm{P} 3$ and $\mathrm{P} 4$ if $\mathrm{O}$ was the cue. We also ran cue neutral. control trials, in which $\mathrm{X}$ preceded compatible stimuli as often as it preceded conflicting stimuli and the same was true of $O$. On these trials, subjects had to attend selectively to the word's identity, employing strategy (1) which uses productions W1 and W2.

In order to assess the time required for strategy construction (i.e., to become prepared to use productions $\mathrm{P} 1$ and $\mathrm{P} 2$ or productions $\mathrm{P} 3$ and P4), we varied the delay between the onset of the cue and the onset of the word. The idea was that reaction time would be faster when subjects had time to construct the strategy than when they did not, so reaction time should decrease as the delay between the cue and the word increased. The delay at which reaction time reached asymptote would give us some idea of the latency of the construction process. We used cue delays of $100,200,400,600,800$, and 1000 msec.

There was no evidence that subjects constructed a strategy when only 100 msec elapsed between the onset of the cue and the word, in that there was no difference between cue-valid and cue-neutral reaction times. However, a difference began to emerge at the longer delays, reaching asymptote between the 400 and $600 \mathrm{msec}$ delays. Thus, in this task, strategy construction was complete in $400-600 \mathrm{msec}$. Subsequent experiments replicated these findings and indicated that strategy construction time could be reduced by practice (see Logan and Zbrodoff (1982) for further details).

Later, we addressed the resource demands of strategy construction using the same task and the same materials. In these experiments, we fixed the delay between the onset of the cue and the word at $600 \mathrm{msec}$ to allow sufficient time for strategy construction, and we presented auditory probes at various times throughout the trial to assess the resource demands of strategy construction. In our first experiment, subjects responded to the probe with the index fingers of their left 
hands, using the index and middle fingers of their right hands to respond to the word. The probe reaction times suggested that strategy construction was very demanding: Probe reaction times were substantially longer when the cue was valid than when it was neutral. The difference was largest when probes were presented immediately after the cue appeared, when subjects were busy constructing the strategy.

These results demonstrate that strategy construction requires resources, but they do not specify which resources it requires. Possibly, general resources are required (i.e., 'central processing capacity'; cf. Posner and Boies 1971) but it is also possible that some very specific resources are required (cf. McLeod 1978). Our second experiment used a different response to the probe in order to discover which kind of resources were required: It was an exact replication of the first experiment, except that subjects shouted $/ \mathrm{ba} /$ into a microphone in response to the probe instead of pressing a key. The pattern of probe reaction times was very different from the pattern in the first experiment: There was very little difference in probe reaction times on cue-valid versus cue-neutral trials, suggesting that strategy construction interfered very little with vocal responses to the probe.

Taken together, the two experiments suggest that the strategy construction observed in this version of the spatial Stroop task requires specific resources rather than general resources. We considered two possibilities: First, strategy construction may involve the resources of the manual motor system, because P1-P4 represent rules for mapping stimuli onto manual motor responses. Possibly, thinking about the manual motor system makes it less available for executing responses until the thinking is complete. Second, strategy construction may involve spatial resources, because P1-P4 represent rules for mapping spatial positions in the display onto spatial positions on the response apparatus. Indeed, manual responses can be thought of as responses to positions in space (e.g., Wickens 1984). Possibly, thinking about space in response to the cue makes it difficult to execute a concurrent response to a position in space (see Logan et al. (1983) for further details).

Our experiments on strategy construction demonstrate that re-configuring the cognitive system takes time and resources, just as other psychological processes take time and resources. Thus, strategy construction can be studied like any other psychological process. Indeed, progress has been made in several laboratories. For example, Dixon 
(1981) found that the time required to set up a stimulus-response mapping rule depends on the number of alternative mappings, and Proctor and Fisicaro (1977) found that setting the system to process different dimensions of a stimulus takes resources. Other examples can be found in the literature on partial advance information (e.g., Welford 1973) and in the literature on motor programming (e.g., Rosenbaum 1980). Nevertheless, the study of strategy construction has only just begun; much remains to be discovered.

\section{Maintaining and executing stratègies}

Once chosen and constructed, a strategy must be executed to perform the task at hand. If the task is a repetitive one (like most tasks in cognitive psychology), the strategy must be maintained from trial. The role of executive functioning in execution is not as clear as it is in choosing and constructing strategies. On the one hand, the strategy may require no further executive intervention during performance, being triggered almost reflexively by an appropriate stimulus and running on to completion ballistically. This possibility has historical roots in the Wurtzburg school's notion of a prepared reflex, and it has been suggested recently as an interpretation of the on-line control of visual search performance (Logan 1978). On the other hand, a strategy may require executive control throughout its execution, requiring a decision about what to do next as each step is completed. This possibility has historical roots as well, and it has also been suggested recently as an interpretation of the on-line control of visual search performance (Schneider and Shiffrin 1977). It is probably the case that some sorts of strategies require executive control throughout their execution while others can run off reflexively. For example, executing a strategy for playing chess may require constant control, but executing the spatial strategy described by Logan and Zbrodoff $(1979,1982)$ may not.

It may prove difficult to determine what sort of control is involved for strategies of intermediate complexity, such as those employed in visual search. Control may be accomplished 'locally' by the special-purpose processes recruited to form the strategy instead of 'centrally' by an executive process. It may be possible to produce a model that assumes local control that mimics the performance of any model that assumes central control, so the issue may turn out to be undecidable. Indeed, students of motor control have debated the issue of central versus 
peripheral control for some time without reaching a satisfactory solution (for a review, see MacKenzie and Marteniuk (1985)). Nevertheless, some progress has been made.

Dual task studies have suggested that the (executive) process of allocating resources consumes resources itself, as if it 'takes attention to pay attention'. For example, Laabs and Stager (1976) showed that a task that required switching attention from one source of information to another interfered more with a concurrent task than a task that required maintaining attention on a single source. Johnston and Heinz (1978) showed that selection on the basis of semantic features interfered more with a concurrent task than selection on the basis of physical features; response set was more demanding than stimulus set. Dividing attention between sources of information or between tasks often produces interference, called concurrence cost, that is not attributable to the demands of either of the sources or tasks. For example, Roediger et al. (1977) found that a Fitts tapping task interfered with short-term retention, but the amount of interference was independent of the difficulty of the tapping task. Duncan (1979) showed convincingly that a substantial amount of the interference between concurrent reaction time tasks was due to emergent interactions between the stimulus-response mapping rules for the tasks that were not present when the tasks were performed alone.

My own research has focused on the maintenance of strategies in working memory. I used a dual-task paradigm in which subjects performed a reaction time task alone or in the retention interval of a short-term memory task. Typically, reaction time is longer with a concurrent memory load than in single-task conditions. I varied parameters of the reaction time task to see which aspects of reaction time performance were responsible for the memory load effect. The idea was that parameters that were responsible for the interference should interact with memory load, whereas parameters that were not responsible should have additive effects (for detailed developments of the arguments, see Logan $(1978,1979,1980 \mathrm{~b}))$. The results suggested that memory load interfered more with factors affecting preparation for the reaction time task than with factors affecting execution. Thus, memory load interacted with the size of the memory set in a Sternberg-type memory search task (Logan 1978: experiments 1 and 2) and with the number of stimulus-response alternatives in a multiple-choice reaction time ask (Logan 1979), and it affected choice reaction time more than 
simple reaction time (Logan 1980b), yet it had additive effects with most other parameters that affect reaction time performance (e.g., visual noise, number of display elements, etc.; see Logan 1978). In other words, memory load interfered most with what subjects had to keep in working memory to perform the tasks (i.e., the rules for mapping stimuli onto responses, which constitute the strategy).

In view of the problems of mimicry mentioned earlier, it may be possible to interpret all of the dual-task results without recourse to executive functioning. The Johnston and Hcinz (1978) results, for example, may reflect the fact that subjects have less information to process in stimulus set than in response set, and the Roediger et al. (1977) findings may indicate that the authors failed to manipulate those aspects of the tapping task that were responsible for the interference with short-term retention (i.e., there may be different kinds of difficulty; see Navon and Gopher 1979; Wickens 1984) or that the Fitts tapping task can be performed relatively automatically once it begins (cf. Naveh-Benjamin and Jonides 1984). My own conclusions about memory load effects were criticized by Gopher and Sanders (1984) [2].

[2] Gopher and Saunders (1984) criticized my work on concurrent memory load, claiming that I had misapplied the additive factors method and that the increase in reaction time with a concurrent memory load may reflect the time required to switch from the memory task to the reaction time task rather than resource competition from concurrent processes. One could argue against Gopher and Sanders' claims about the appropriateness of the additive factors logic, but even if their arguments are correct, it is important to note that I used the additive factors logic only in the 1978 paper. The 1979 paper on multiple-choice reaction time and the $1980 \mathrm{~b}$ paper on simple and choice reaction time explicitly avoided the use of the additive factors logic. Indeed, the points made in the three papers could be made without reference to the additive factors mcthod; the experiments were designed to discover the parameters of reaction time tasks that were influenced by concurrent memory load. Whether those parameters effect specific stages of processing is another question entirely.

Gopher and Sanders' second criticism, that my effects reflect the time required to switch from the memory task to the reaction time task, cannot account for my data. The idea of switching cannot account for the fact that some parameters interacted with concurrent memory load (e.g., memory set size, number of stimulus-response alternatives, simple vs choice tasks) and others didn't (e.g., visual noise, array size, presence vs absence of a bar marker, yes vs no decision, stimulus-response compatibility; see Logan (1978)). Subjects should switch in all conditions, regardless of the parameters that are being manipulated. Thus, interactions should be observed in all conditions or additive effects should be observed in all conditions, yet the data showed a (readily interpretable) mixture of interactions and additive effects. Furthermore, the idea of switching cannot account for the practice effects I observed: Practice with consistent mapping eliminated interactions between concurrent memory load and memory set size (Logan 1978: experiment 1 ) and between concurrent memory load and number of stimulus-response alternatives (Logan 1979: experiment 1); changing the mappings on the seventh session caused the interactions 
Nevertheless, the possibility that these effects reflect executive functioning cannot be ruled out entirely. The idea that 'it takes attention to pay attention' is intriguing, and promises to be a fruitful direction for future research.

Two current lines of research seem to promise new insight into on-line executive control. One is Pat Rabbitt's analysis of subjects' control of speed and accuracy, in which subjects are thought to adjust criteria for speed and accuracy in response to judgements about the adequacy of their performance, speeding up if they are too slow and slowing down if they make and error (see e.g., Rabbitt 1981). The second is James Reason's analysis of action slips and everyday errors, in which qualitative aspects of the errors people make provide insight into what they intended to do and what they tried to control in carrying out their intentions (sec c.g., Reason 1984). In contrast with the dual-task studies mentioned above that tried to assess the amount of control with a unidimensional interference measure, Rabbitt and Reason have tried to specify in detail what is being controlled and what people do to achieve control. The more detailed analysis seems more realistic and may prove to be less susceptible to alternative interpretation (cf. Jonides et al. 1985). In any event, the investigation executive control has only just begun. The field is still wide open, and much important work remains to be done.

\section{Disengaging strategies}

After choosing, constructing, and executing a strategy, people must eventually disengage it. Sometimes, the task will have finished; other times the goal will no longer be relevant; still other times the person will have made an error that needs to be corrected. In each case, the strategy must be disabled or disengaged so the person can move on to a new task. The process of disengagement can be studied like the other executive functions, in terms of the time it takes and the resources it

to return in both of these experiments; and practice with varied mapping failed to eliminate interactions between memory load and memory set size (Logan 1978: experiment 2 ) and between concurrent memory load and number of alternatives (Logan 1979: experiment 2). These effects seem more consistent with the interpretation that I offered, namely, that a concurrent memory load interferes with subjects' ability to maintain the instructed mapping rules in working memory (see Logan 1978, 1979, 1980b). 
consumes. In addition, we can ask whether there is any 'inertia' to the current strategy, assessing the extent to which ongoing processes run on to completion ballistically. Each of these questions can be addressed to the overt behavior that results from the strategy or to the thoughts that underly it.

\section{Stopping action}

Current research suggests that strategies can be disengaged from motor systems in about $200 \mathrm{msec}$ (for a review, see Logan and Cowan (1984)). This is apparent in studies of spontaneous and instructed error detection: Levelt (1983) noted that adult speakers stopped speaking spontaneously within one or two syllables of making an error. Similarly, Long (1976) and Rabbitt (1978) instructed skilled typists to stop typing when they made an error and observed that they stopped typing within one or two keystrokes of making an error. It was possible to estimate the time required to detect an error in Rabbitt's typing study by multiplying the mean number of characters typed after an error by the mean typing rate, which yielded an estimate of $182 \mathrm{msec}$.

However, error detection studies may underestimate the time required to disengage an action plan. Subjects may sometimes detect an error before they actually make it (Rabbitt 1978), so the stopping latency could be substantially longer than the $182 \mathrm{msec}$ estimated above (i.e., it could include the $182 \mathrm{msec}$ plus the time to make the erroneous movement, which may be as much as $250 \mathrm{msec}$; see Gentner et al. 1980). Also, subjects may know when they are likely to make an error and they may adopt a different mode of control to deal with it, perhaps being more vigilant than they might be otherwise. These problems can he overcome by presenting subjects with an overt signal to stop (e.g., a tone), which allows the experimenter to know exactly when the stopping process begins but prevents the subject from anticipating the need to stop.

Stop-signal studies of speech and typing corroborate the error detection studies in suggesting that skilled speakers and typists can inhibit their actions relatively quickly. Ladefoged et al. (1973) found that subjects stopped speaking about $200 \mathrm{msec}$ after the stop signal occurred, and Logan (1982) found that subjects stopped typing about 300 msec after the stop signal occurred.

The stop-signal and error-detection studies suggest that people can 
disengage strategies very quickly; typists and speakers seem capable of stopping in the middle of a word, as if only very small units of action were ballistic. However, this rapid disengagement need not be interpreted as reflecting typical on-line control, as if typists and speakers programmed very small units of action. It may be more appropriate to interpret it as a high-level (executive) intervention, intended to deal with a 'crisis' initiated by an error or a change in goals (i.e., stop rather than go). Its relevance to the normal mode of control may be to suggest that control may be hierarchical: Possibly, skills like speech and typing are controlled locally from second to second, with an executive process overseeing the stream of action, ready to intervene should it seem necessary (cf. Logan and Cowan 1984). By analogy, thought and action may be controlled like a factory, with individual workers and foremen controlling on-line production, overseen by the boss who monitors their performance, intervening occasionally to keep it in line with the broader goals of the company.

The impressive speed with which actions can be stopped implies that the response to a stop signal or an error is not subject to the same resource limitations as overt responses to similar signals. Indeed, the response to a stop signal seems to take about the same amount of time regardless of the complexity of the task being stopped (for a review, see Logan and Cowan (1984)). For example, Logan et al. (1984a) found that subjects took only $17 \mathrm{msec}$ more to stop a choice reaction time response than to stop a simple reaction time response. One way to interpret these findings is that an executive process may evaluate the significance of incoming stimuli, responding to them quickly if they signify changes in goals or placing them in a queue with the other responses if they require an overt response. Thus, the response to a stop signal or an error may compete for resources but it may have such high priority that it always wins (Logan and Cowan (1984).

\section{Stopping thought}

People also need to stop or change their current course of thought when it is finished or no longer relevant to current goals. This kind of executive intervention can also be studied with the stop signal method, but the application is more complicated because thoughts are more difficult to observe than overt actions. Cognitive psychologists typically observe thought by coupling some action with thought (e.g., 'decide 
whether "professor" is a profession and press one of two buttons to indicate your decision'), and basing inferences about the underlying thought on observable properties of the response (i.e., its accuracy and latency). However, if subjects are asked to inhibit the responses that arc coupled with the underlying thought, the major index of thought is lost; there is no response whose accuracy and latency can be observed. Instead, thoughts can be made observable through their aftereffects, such as memory, repetition, and priming effects, and the magnitude of the aftereffects of thoughts that might have been inhibited can be compared with the magnitude of the aftereffects of thoughts that were likely to have gone on the completion: If the thoughts were inhibited, the aftereffects should be weaker than those of thoughts than ran on to completion.

I used this logic to assess the inhibition of thought in category and rhyme judgement tasks (see Logan 1983, 1985). Subjects were given an orienting phase, in which they saw pairs of words and made category and rhyme judgements about them (e.g., is a frigate a boat?, does sleigh rhyme with play?). On $30-40 \%$ of the trials a stop signal sounded, and subjects were told to inhibit their overt responses when they heard the signal. Subjects were then given a memory test, in which they were presented with words that had appeared in the orienting phase with a stop signal (stop-signal words), words that had appeared in the orienting phase without a stop signal (no-signal words), and words that had not appeared in the orienting phase (new words). In one set of experiments (Logan 1983), the memory test assessed subjects' ability to recognize the words; in another set of experiments (Logan 1985), the memory test assessed repetition priming (i.e., the extent to category and rhyme judgments were faster for stop-signal and no-signal words that had been seen before than for new words that had not been seen before).

In both sets of experiments, memory performance was assessed as a function of stop-signal delay in order to determine whether the underlying thoughts went on to completion when the overt actions were inhibited. The idea was that thoughts were more likely to have been inhibited at the early delays than at the late delays because the corresponding actions were in fact inhibited more often at the early delays than at the late delays. Thus, if the underlying thoughts were inhibited with the overt actions, then memory performance should improve as stop signal delay increased, going from near-chance levels at 
the early delays to the level of performance for no-signal words at later delays. However, if the underlying thoughts went on to completion whether or not the overt actions were inhibited, then memory performance should not be affected by stop-signal delay, being close to performance for no-signal words at all delays.

When subjects had only to stop their responses when the stop signal sounded, the underlying thoughts seemed to run on to completion whether or not the accompanying action was inhibited. Recognition accuracy was unaffected by stop-signal delay (Logan 1983; experiments 1 and 2) as was the amount of repetition priming (Logan 1985: experiment 1). When subjects had to stop their responses and switch to a new task (responding to the tone), the underlying thoughts still seemed to run on to completion. Recognition accuracy was unaffected by stop signal delay (Logan 1983: experiments 3 and 4), and repetition priming was only slightly affected (Logan 1985: experiment 2). The only procedure that seemed to inhibit the underlying thought was to terminate the display of the word pair and replace it with a new one. This produced a substantial effect of stop-signal delay on recognition accuracy, going from chance levels at the shortest delay to the level of no-signal performance at the longest delay (Logan 1983: experiments 5 and 6). It also produced a substantial effect of stop-signal delay on repetition priming, going from chance levels at the earliest delay (i.e., no difference in reaction time to new vs repeated words) to the level of no-signal performance at the longest delay (Logan 1985: experiment 4). The thought inhibition was not entirely due to a masking effect from the stimulus that replaced the word to be judged, since a control experiment in which the display changed but subjects were told not to inhibit their responses produced a much weaker effect of stop-signal delay (Logan 1985: experiment 5).

Taken together, these results suggest that the simple thoughts underlying category and rhyme judgments will run on to completion unless the stimuli that drive them are disrupted. More generally, these experiments demonstrate that is is possible to observe the inhibition of thought, and thus, to observe the process of disengaging strategies that do not involve overt action. The experiments open up new lines of enquiry, raising many important questions. Much research remains to be done. 


\section{Conclusions and implications}

This paper has selectively reviewed studies of four major executive functions. The review supports several conclusions: First, it demonstrates that executive functions have empirical consequences in the kinds of tasks commonly studied in cognitive psychology. Thus, if we hope to understand the tasks we already study, we must learn about the executive functions that control and coordinate them. Second, the review demonstrates that executive functions can be studied by applying the methods and analyses we already use to study other psychological functions. Much of the technology needed to understand executive functioning already exists; we need only to apply it. Third, the review demonstrates that we know very little about executive functioning. In addition to the work on basic information processing reviewed here, students of higher-level cognition have worked on theories of control in complex tasks (see e.g., the production system theories of Anderson (1983)) and developmental psychologists have addressed children's control over memory processes (see e.g., metacognition and metamemory discussed by Flavell and Wellman (1977)). Nevertheless, few psychologists pay much attention to the strategic aspects of cognition and to problems of coordination and control. Much important work remains to be done.

There are two major directions for future work to take: First, the empirical picture presented in this review can provide no more than a rough sketch of the basic phenomena. More of the details need to be filled in. For example, what other factors besides cue validity and resource requirements affect choice among strategies? What factors affect the time required to construct a strategy? How is strategy construction affected if one strategy must be disengaged before another can be constructed? More generally, are the executive functions I distinguished really separate? Are there more executive functions besides the four reviewed here? Are all the executive functions achieved by a single executive process, or are there several executives? Second, the empirical picture needs to be supplemented by sound theory. It would be very useful to have enumerated the elementary psychological processes that form the basis of strategies and it would be useful to understand the rules by which elementary processes can be combined to form strategies. Mewhort and Campbell (1981) have begun to develop a theory of this sort to describe the strategic options available in early 
visual information processing. Others need to follow in their footsteps so that the study of executive functioning can be guided by logical principles as well as psychologists' intuitions.

\section{References}

Anderson, J.R., 1983. Architecture of cognition. Cambridge, MA: Harvard University Press.

Dixon, P., 1981. Algorithms and selective attention. Memory and Cognition 9, 177-184.

Duncan, J., 1979. Divided attention: the whole is more than the sum of the parts. Journal of Fxperimental Psychology: Human Perception and Performance 5, 216-228.

Flavell, J.H. and H.M. Wellman, 1977. 'Metamemory'. In: R. Kail and J.W. Hagen (eds.), Perspectives on the development of memory and cognition. Hillsdale, NJ: Erlbaum.

Gentner, D.R., J.T. Grudin and E. Conway, 1980. Finger movements in transcription typing (Tech. Rep. 8001). La Jolla, CA: University of California at San Deigo, Center for Human Information Processing.

Gopher, D. and A.F. Sanders, 1984. 'S-oh-R: Oh, stages; oh resources'. In: W. Prinz and A.F. Sanders (eds.), Cognition and motor processes. Berlin: Springer.

Johnston, W.A. and S.P. Heinz, 1978. Flexibility and capacity demands of attention. Journal of Experimental Psychology: General 107, 420-435.

Jonides, J., M. Naveh-Benjamin and J. Palmer, 1985. Assessing automaticity. Acta Psychologica $60,157-171$.

Laabs, G.J. and P. Stager, 1976. Monitoring the information-processing demands of attention switching. Canadian Journal of Psychology 30, 47-54.

Ladefoged, P., R. Silverstein and G. Papcun, 1973. Interruptibility of speech. Journal of the Acoustical Society of America 54, 1105-1108.

Levelt, W.J.M., 1983. Monitoring and self-repair in speech. Cognition 14, 41-104.

Logan, G.D., 1978. Attention in character classification: evidence for the automaticity of component stages. Journal of Experimental Psychology: General 107, 32-63.

Logan, G.D., 1979. On the use of a concurrent memory load to measure attention and automaticity. Journal of Experimental Psychology: Human Perception and Performance 5, 189-207.

Logan, G.D., 1980a. Attention and automaticity in Stroop and priming tasks: theory and data. Cognitive Psychology 12, 523-553.

Logan, G.D., 1980b. Short-term memory demands of reaction-time tasks that differ in complexity Journal of Experimental Psychology: Human Perception and Performance 6, 375-389.

Logan, G.D., 1982. On the ability to inhibit complex movements: a stop-signal study of typewriting. Journal of Experimental Psychology: Human Perception and Performance 8, 778-792.

Logan, G.D., 1983. On the ability to inhibit simple thoughts and actions: I. Stop-signal studies of decision and memory. Journal of Experimental Psychology: Learning, Memory and Cognition 9, 585-606.

Logan, G.D., 1985. On the ability to inhibit simple thoughts and actions: II. Stop-signal studies of repetition priming. Journal of Experimental Psychology: Learning, Memory and Cognition 11, $675-691$

Logan, G.D. and W.B. Cowan, 1984. On the ability to inhibit thought and action: a theory of an act of control. Psychological Review 91, 295-327.

Logan, G.D. and N.J. Zbrodoff, 1979. When it helps to be misled: facilitative effects of increasing the frequency of conflicting stimuli in a Stoop-like task. Memory and Cognition 7, 166-174. 
Logan, G.D. and N.J. Zbrodoff, 1982. Constraints on strategy construction in a speeded discrimination task. Journal of Experimental Psychology: Human Perception and Performance 8, $502-520$.

Logan, G.D., N.J. Zbrodoff and A.R. Fostey, 1983. Costs and benefits of strategy construction in a speeded discrimination task. Memory and Cognition 11, 485-493.

Logan, G.D., W.B. Cowan and K.A. Davis, 1984a. On the ability to inhibit simple and choice reaction time responses: a model and a method. Journal of Experimental Psychology: Human Perception and Performance 10, 276-291.

Logan, G.D., N.J. Zbrodoff and J. Williamson, 1984b. Strategies in the color-word Stroop task. Bulletin of the Psychonomic Society 22, 135-138.

Long, J., 1976. Visual feedback and skilled keying: differential effects of masking the printed copy and the keyboard. Ergonomics 19, 93-110.

MacKenzie, C.L. and R. Marteniuk, 1985, Motor skill. Canadian Journal of Psychology 39, 313-337.

McLeod, P.D., 1978. Does probe RT measure central processing demand? Quarterly Journal of Experimental Psychology 30, 83-89.

Mewhort, D.J.K. and A.J. Campbell, 1981. 'Toward a model of skilled reading: an analysis of performance in tachistoscopic tasks'. In: G.E. MacKinnon and T.G. Waller (eds.), Reading research: advances in theory and practice, Vol. 3. Academic Press. pp. 39-118.

Naveh-Benjamin, M. and J. Jonides, 1984. Maintenance rehearsal: a two-component analysis. Journal of Experimental Psychology: Learning, Memory and Cognition 10, 369-385.

Navon, D. and O. Gopher, 1979. On the economy of the human processing system. Psychological Review 86, 214-255.

Neely, J.H., 1977. Semantic priming and retrieval from lexical memory: roles of inhibitionless spreading activation and limited-capacity attention. Journal of Experimental Psychology: General 106, 226-254.

Posner, M.I. and S.J. Boies, 1971. Components of attention. Psychological Review 78, 391-408.

Posner, M.I. and C.R.R. Snyder, 1975. 'Attention and cognitive control'. In: R.L. Solso (ed.), Information processing and cognition: the Loyola symposium. Hillsdale, NJ: Erlbaum.

Proctor, R.W. and S.A. Fisicaro, 1977. Time, capacity, and selection between perceptual attributes. Journal of Experimental Psychology: Human Perception and Performance 3, 337-348.

Rabbitt, P.M.A., 1978. Detection of errors by skilled typists. Ergonomics 21, 945-958.

Rabbitt, P.M.A., 1981. 'Sequential reactions'. In: D.H. Holding (ed.), Human skills. New York: Wiley.

Reason, J., 1984. 'Lapses of attention in everyday life'. In: R. Parasuraman and D.R. Davies (eds.), Varieties of attention. New York: Academic Press.

Roediger, H.L., S.L. Knight and B.H. Kantowitz, 1977. Inferring decay in short-term memory: the issue of capacity. Memory and Cognition 5, 167-176.

Rosenbaum, D.A., 1980. Human movements initiation: specification of arm, direction, and extent. Journal of Experimental Psychology: General 109, 444-474.

Schneider, W. and R.M. Shiffrin, 1977. Controlled and automatic human information processing: I. Detection, search, and attention. Psychological Review 84, 1-66.

Tweedy, J.R., R.H. Lapinski and R.W. Schvaneveldt, 1977. Semantic context effects on word recognition: influence of varying the proportion of items presented in an appropriate context. Memory and Cognition 5, 84-89.

Welford, A.T., 1973. 'Attention, strategy and reaction time: a tentative metric'. In: S. Kornblum (ed.), Attention and performance IV. New York: Academic Press.

Wickens, C.D., 1984. 'Processing resources in attention'. In: R. Parasuraman and D.R. Davics (eds.), Varieties of attention. New York: Academic Press.

Zbrodoff, N.J. and G.D. Logan, in press. On the autonomy of mental processes: a case study of arithmetic. Journal of Experimental Psychology: General. 